\title{
Primary Effusion Lymphoma in a patient with a Good Outcome Despite the Lack of Chemotherapy
}

\author{
Ofir Koren ${ }^{1}$, Ariel Aviv ${ }^{1}$, Moran Avraham Kelbert ${ }^{1}$, Ehud Rozner ${ }^{1}$, Liza Lihtman ${ }^{1}$, and \\ Yoav Turgeman ${ }^{1}$ \\ ${ }^{1}$ Emek Medical Center
}

October 28, 2020

\begin{abstract}
Background Primary effusion lymphoma (PEL) is a rare disease occurs mostly in immunosuppressed young males. Treatment consists of chemotherapy and prognosis is poor. Case presentation Our case described immunocompetent elderly woman with PEL who survive 30 months without chemotherapy. Conclusion Physicians should be aware of all clinical scenarios of PEL

Primary Effusion Lymphoma in a patient with a Good Outcome Despite the Lack of Chemotherapy

Ofir Koren MD, FICA ${ }^{1,4}$, Ariel Aviv $\mathrm{MD}^{2,4}$, Moran Avraham Kelbert $\mathrm{PhD}^{3}$,

Ehud Rozner $\mathrm{MD}^{1}$, Liza Lihtman $\mathrm{BA}^{1}$, Elya Halfin $\mathrm{MD}^{4}$, Yoav Turgeman $\mathrm{MD}^{1,4}$

${ }^{1}$ Heart Institute, Emek Medical Center, Afula, Israel

${ }^{2}$ Hematology Department, Emek Medical Center, Afula, Israel

${ }^{3}$ Department of laboratory medicine, Emek Medical Center, Afula, Israel

${ }^{4}$ Bruce Rappaport Faculty of Medicine, Technion-Israel Institute of Technology, Haifa, Israel
\end{abstract}

\section{Corresponding Author:}

Ofir Koren, MD, FICA

Drkorenofir@gmail.com

Tel: $972-46495121$

Fax: 972-48141414

Address : Emek Medical Center Rabin Boulevard 21, Afula, Israel 1834111

This author takes responsibility for all aspects of the reliability and freedom from bias of the data presented and their discussed interpretation

Running Title : Primary Effusion Lymphoma in Non-Immunocompromised Patient

Word count: Abstract (50), Manuscript (1662)

\section{Background}

Primary effusion lymphoma (PEL) is a rare disease occurs mostly in immunosuppressed young males. Treatment consists of chemotherapy and prognosis is poor. 


\section{Case presentation}

Our case described immunocompetent elderly woman with PEL who survive 30 months without chemotherapy.

\section{Conclusion}

Physicians should be aware of all clinical scenarios of PEL.

\section{Key Clinical Message}

Primary effusion lymphoma mostly occurs in immunosuppressant young males. The overall prognosis is poor. We highlight a case of PEL in an immunocompetent HIV-negative elderly woman who had a good outcome despite the lack of chemotherapy treatment.

\section{KEYWORDS}

Human Immunodeficiency Virus; Primary Effusion Lymphoma; Pericardial Effusion, Pleural Effusion, Human Herpes Virus type 8.

\section{ABBREVIATION}

PEL, Primary Effusion Lymphoma; HHV8, Human Herpes Virus type 8; HIV, Human Immunodeficiency Virus; EBV, Epstein-Bar Virus; CT, computerized tomography, ECG, electrocardiography; NHL, NonHodgkin's lymphoma; DLBCL, diffuse large B-cell Lymphoma. LLL, left lower lobe.

\section{BACKGROUND}

Primary effusion lymphoma (PEL) is a rare and distinct type of high-grade non-Hodgkin's B-cell lymphoma (NHL) [1]. Human herpesvirus type 8 (HHV8) been directly linked to PEL since the late 1990s [2].

PEL is characterized by the presence of significant serous lymphomatous fluid collection in the pleural, pericardial, and peritoneal cavities without detectable solid tumor masses [3].

It most often occurs in immunocompromised patients such as in the advanced stages of Acquired Immune Deficiency Syndrome (AIDS) [4]. PEL has also been reported in immunocompetent elderly patients from HHV-8 endemic areas such as central and southern Africa and the Mediterranean area. It typically involves Middle-aged patients with a significant male predominance of $6: 1$ [5].

The clinical scenario is directly related to the body cavity involved, the amount of the effusion, and its interaction with the adjacent structures [6].

The diagnosis of PEL is based on cytological immunophenotypically and viral characteristics of the involved effusion. PEL is suspected in the presence of diffuse large cells with abundant basophilic cytoplasm and irregular nuclei with prominent nucleoli resembling plasma cells [7].

The lymphoma cells usually express the leukocyte origin marker CD45 but lack the typical B or T cell phenotype while markers of plasma cell differentiation, CD38, and CD138 are usually present [8].

Uniform treatment of PEL has not been properly established, and patients are generally treated with combined chemotherapy used for aggressive lymphomas known as the "CHOP" protocol, consisting of cyclophosphamide, hydroxydaunorubicin, oncovin (vincristine) and prednisone. Despite complete response rates of 43 to $57 \%$, the median survival is still poor and estimated as six to nine months, due to early and usually refractory relapses [9].

\section{CASE PRESENTATION}

An 84-years old Jewish woman of North African ancestry presented to the emergency room complaining of effort-related dyspnea, low-grade fever, and a dry cough for several days. her past medical history includes only controlled hypertension. She uses Cinnarizine, Nifedipine, Aspirin, and Atenolol on a regular basis. 
Upon arrival, Chest X-ray (Figure 1) showed an enlarged heart silhouette and left lower lobe (LLL) infiltrate. Physical examination revealed diminished breath sounds and pleural dullness during percussion of the left hemithorax.

After several days with no meaningful clinical improvement following treatment with azithromycin and ceftriaxone, CT angiography was performed indicating significant bilateral pleural effusion, moderate pericardial effusion and no lymphadenopathy.

Transthoracic echocardiography (Figure 2) showed a normal function of the left ventricle and large volume of pericardial effusion with a diastolic right-sided collapse. Urgent pericardiocentesis via a subcostal approach was performed and $650 \mathrm{ml}$ of serosanguinous fluid were withdrawn resulting in an immediate relief of symptoms. Pericardial drainage continued for nine days using a pigtailed catheter.

The patient underwent left pleurocentesis with the removal of $500 \mathrm{ml}$ of exudative cloudy fluid. Pleural cell count indicated a total of $42,700 \mathrm{k} / \mathrm{ul}$ of white blood cells. Of them, $24 \%$ were monocytes, $13 \%$ basophils, and $63 \%$ were large unstained cells [LUC]. Pleural chemistry analysis showed a significant low glucose level $[<11 \mathrm{mg} / \mathrm{dL}]$, high protein level [4.36 g/dL], Low pH [6.99], and an extremely high LDH level [44,000, U/L] while Serum LDH was mildly elevated $[640 \mathrm{U} / \mathrm{L}]$. The effusion to serum protein ratio was 0.71 .

Flow cytometry (NAVIOS machine Beckman Coulter, USA), from the pericardial fluid, analyzed using the side scatter (SSC) gating, indicated 100,000 white blood cells. Of which, $25 \%$ were neutrophils $(\mathrm{CD} 13+/ \mathrm{CD} 10+), 25 \%$ monocytes (CD64+, CD123-, CD33+) and $12 \%$ were from the lymphoid lineage (CD20+, CD3+). An additional CD45- population comprised 38-45\% of total cells with the following antigen properties: Kappa+, Lambda-, CD138+, CD38+, CD56+, CD81+, CD229+, CD74+, CD43+, CD19-, CD20-, CD24-, CD54-, CD27-, CD28-, CD200dim, CD117-, CD10-. CD81+ (Figure 3).

Microscopic examination of the fluid revealed large immature atypical lymphocyte. Broad antigens analysis was performed indicating the following: HLA-DR-, CD34-, CD123-, CD33-, CD64-, CD68-, CD4-, cyCd79a-, cyCD3-, MPO-, TdT-.

Flow cytometry of the Pleural fluid showed the same antigenic properties (Figure 4). Immunofluorescent assays (IFA) using the lytic and latent IgG test for the detection of HHV-8 were both positive. Human immunodeficiency virus (HIV) testing using the enzyme-linked immunosorbent assay was negative.

A diagnosis of primary effusion lymphoma was established based on the cytologic findings. suggestive of a lymphoid malignant infiltrate with cells positive for HHV8, along with the peculiar characteristic immunophenotype which was negative for classical B and $\mathrm{T}$ cell markers, yet positive for plasma cell markers i.e CD38 and CD138. Additionally, the lack of lymph node or spleen enlargement in whole body CT, and in fact - the lack of any masses at all were all consistent with the diagnosis of PEL.

After consulting with her family members, the patient chose not to start chemotherapy. Her treatment regimen consisted of anti-hypertensive medication (Nifedipine and Bisoprolol), 60mg Prednisone which was slowly tapered over a course of several week, Ibuprofen (1800mg daily for two weeks), 30mg Lansoprazole and 100mg Aspirin.

During the first six months after discharge, she underwent two successful pleural drains. At a two-and-ahalf-year follow-up there were no adverse events other than a mild accumulation of pleural fluid that did not require drainage.

\section{DISCUSSION}

Primary effusion lymphoma is a rare lymphoma that usually occurs in immunosuppressed middle-aged males. Recent reports also described PEL in immunocompetent HIV-negative patients, mainly associated with HHV-8 infection in endemic areas. The most widely used protocol is the intensive chemotherapy combination known as the CHOP protocol, designed for aggressive lymphoma. It is accompanied by serious adverse side effects, a low remission rate, and short duration of remissions in those who do respond. 
In most reports, the estimated life expectancy of immunocompromised PEL patients is of a few months, commonly reported as six to nine months. The reason probably relates to the progressive nature of the disease, patients comorbidities, compromised immune system status, procedural complications, failure of treatment and adverse effect of chemotherapy.

Our case highlights an extremely rare scenario of PEL that occurred in an immunocompetent HIV-negative elderly female in a non HHV8-endemic area. PEL, as seen in our case, involved both the pleural and the pericardial spaces. Despite the patient's advanced age, her overall physical and cognitive function were intact. The patient chose not to be treated with chemotherapy and her sole treatment was prednisone. Over a course of 30 months, there were no adverse events. The patient is still alive and in complete remission and maintains an excellent quality of life.

\section{CONCLUSION}

It seems that PEL has different clinical scenarios which we still don't fully understand affecting both immunocompetent and immunosuppressed patients, each with different epidemiologic features and different prognosis. The role of chemotherapy treatment in our case is still to be discovered as the patient decided not to abstain from active treatment. Physicians should be aware of all clinical scenarios to offer the best treatment option for their patients.

\section{DECLARATIONS}

- Consent for publication - Written informed consent was obtained from the patient for publication of this case report and any accompanying images.

- Availability of data and materials - The datasets used and/or analyzed during the current study are available from the corresponding author on reasonable request

- Competing interests - The authors have no conflicts of interest to declare

- Funding - The authors have not declared a specific grant for this research from any funding agency in the public, commercial, or not-for-profit sectors.

- Authors' contributions -OK, AA and MAK contributed to the writing, editing, formatting of the main manuscript, and production of the figures. EP, LL, and YT provided care to the patient and revised the manuscript. All authors have contributed and met the criteria for authorship.

- Acknowledgments - We would like to thank Izabel Ben Guy for her help and contribution to the research

\section{REFERENCE}

1. Carbone A, Gloghini A. PEL and HHV8-unrelated effusion lymphomas: classification and diagnosis. Cancer . 2008;114(4):225-227. doi:10.1002/cncr.23597

2. Fassone L, Bhatia K, Gutierrez M, et al. Molecular profile of Epstein-Barr virus infection in HHV-8positive primary effusion lymphoma. Leukemia . 2000;14(2):271-277. doi:10.1038/sj.leu.2401651

3. Chen J, Mehraj V, Szabo J, Routy B, Michel RP, Routy JP. Multiple remissions of extracavitary primary effusion lymphoma treated with a single cycle of liposomal doxorubicin in a patient infected with HIV. Curr Oncol. 2018;25(6):e592-e596. doi:10.3747/co.25.4119

4. Ota Y, Hishima T, Mochizuki M, et al. Classification of AIDS-related lymphoma cases between 1987 and 2012 in Japan based on the WHO classification of lymphomas, fourth edition. Cancer Med . 2014;3(1):143-153. doi:10.1002/cam4.178

5. Li S, Bai L, Dong J, Sun R, Lan K. Kaposi's Sarcoma-Associated Herpesvirus: Epidemiology and Molecular Biology. Adv Exp Med Biol . 2017;1018:91-127. doi:10.1007/978-981-10-5765-6_7

6. Narkhede M, Arora S, Ujjani C. Primary effusion lymphoma: current perspectives. Onco Targets Ther . 2018;11:3747-3754. Published 2018 Jun 28. doi:10.2147/OTT.S167392

7. Lurain K, Polizzotto MN, Aleman K, et al. Viral, immunologic, and clinical features of primary effusion lymphoma. Blood . 2019;133(16):1753-1761. doi:10.1182/blood-2019-01-893339

8. Fan W, Bubman D, Chadburn A, Harrington WJ Jr, Cesarman E, Knowles DM. Distinct subsets of primary effusion lymphoma can be identified based on their cellular gene expression profile and viral 
association. J Virol . 2005;79(2):1244-1251. doi:10.1128/JVI.79.2.1244-1251.2005

9. Arora N, Gupta A, Sadeghi N. Primary effusion lymphoma: current concepts and management. Curr Opin Pulm Med . 2017;23(4):365-370. doi:10.1097/MCP.0000000000000384

\section{FIGURES \& LEGENDS}

Figure 1. Chest X-ray On admission

Figure 2. Transthoracic Echocardiography (TTE) on admission

Figure 3. Pericardial Flow Cytometry

Figure 4. Pleural Flow Cytometry

Figure 1. Chest X-ray on admission shows enlargement of the cardiac silhouette and large left pleural effusion (yellow arrow)

Figure 2. Transthoracic Echocardiography (TTE) on admission indicates large pericardial effusion (yellow arrow) seen on subcostal view (figure A) and Short axis view (figure B).

Figure 3. Eight color flow cytometric immunophenotyping of pericardial fluid indicated 34\% CD45 negative cell population (A). Representative dot plots gated on CD45- (K gate) cells show high expression of CD138 (B), negative expression of CD27 (C), CD28 (D), CD19 (F) and a partial expression of CD81 (E). CD45cells show positive monoclonality for Kappa light chain $(\mathrm{G})$.

Figure 4. Eight color flow cytometric immunophenotyping of pleural fluid indicated $46 \%$ CD45 negative cell population (A and B). Representative dot plots gated on CD45- (K gate) cells show high expression of CD138 (C) and a partial expression of CD81 (D).

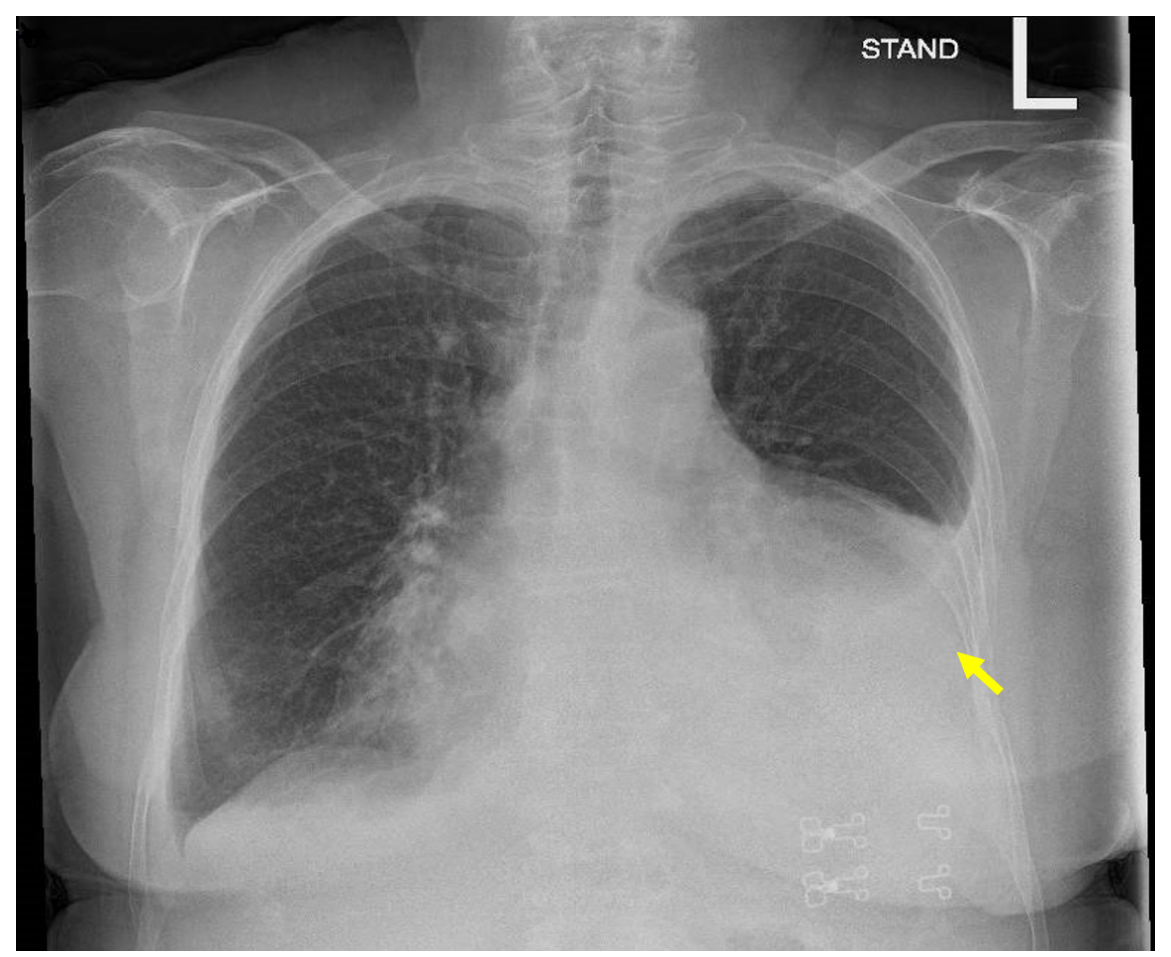



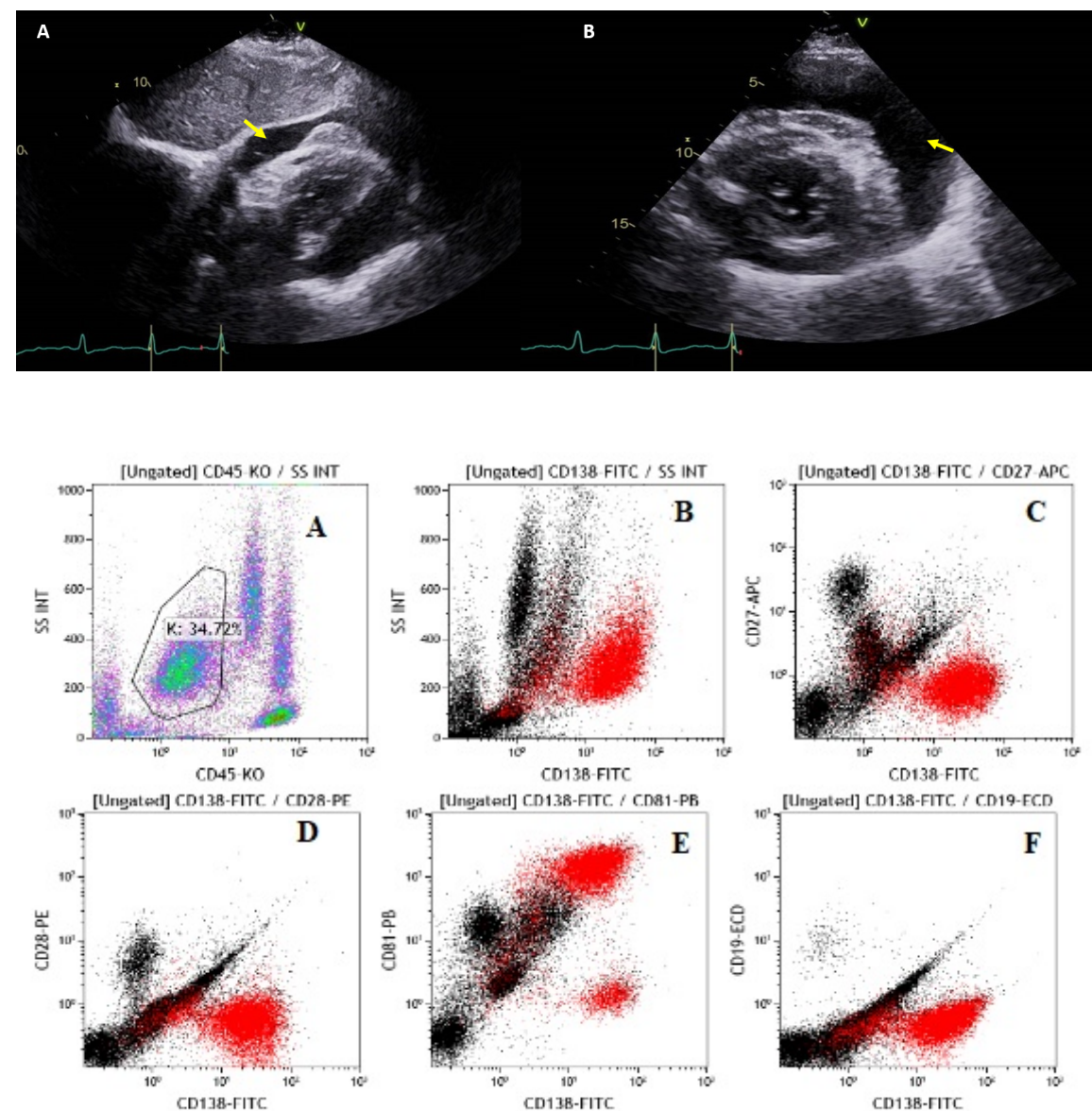

FS INT

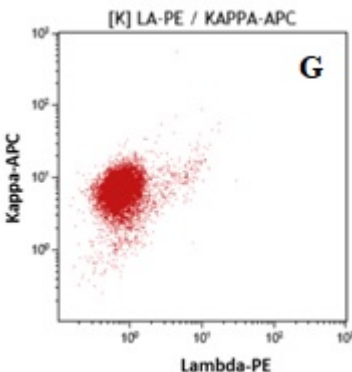



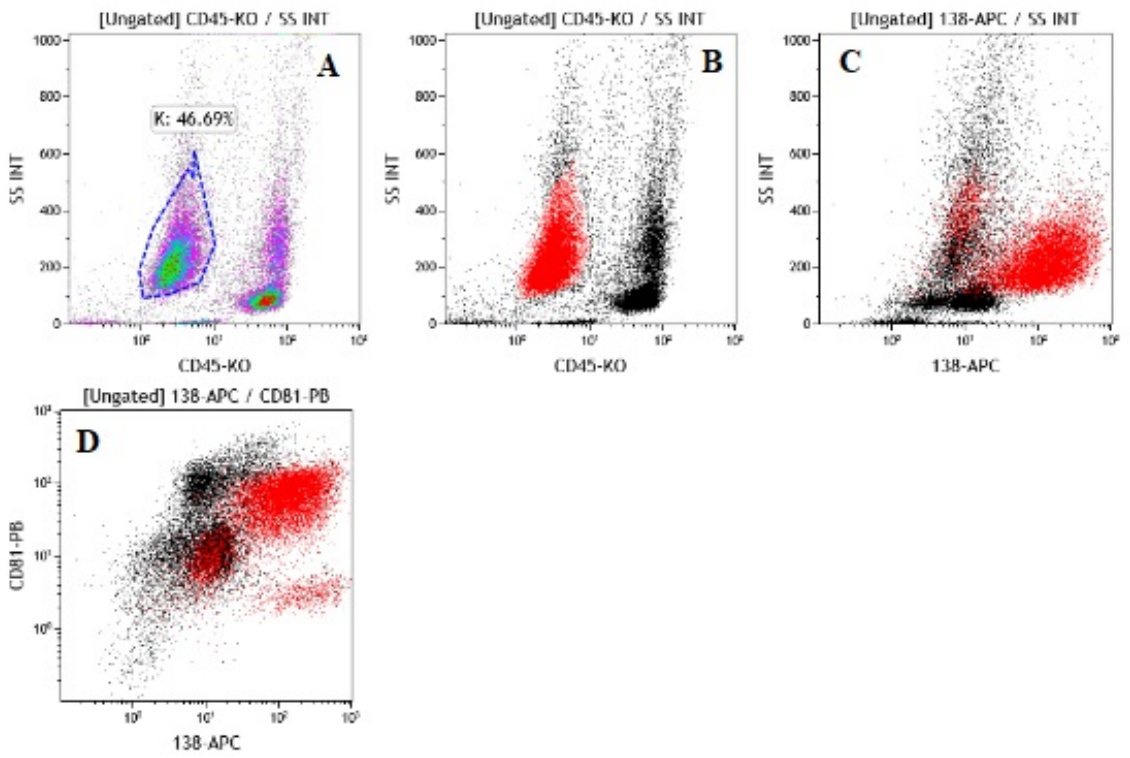